\title{
Group analysis in the SSRS2 catalog
}

\begin{abstract}
C. Adami and A. Mazure
LAM, Traverse du Siphon, 13012 Marseille, France

Received 31 July 2001 / Accepted 24 October 2001

Abstract. We present an automated method to detect populations of groups in galaxy redshift catalogs. This method uses both analysis of the redshift distribution along lines of sight in fixed cells to detect elementary structures and a friend-of-friend algorithm to merge these elementary structures into physical structures. We apply this method to the SSRS2 galaxy redshift catalog. The groups detected with our method are similar to group catalogs detected with pure friend-of-friend algorithms. They have similar mass distribution, similar abundance versus redshift, a similar 2-point correlation function (modeled by a power law: $\left(r / r_{0}\right)^{\gamma}$ with $r_{0}=7 h^{-1} \mathrm{Mpc}$ and $\gamma=-1.79)$ and the same redshift completeness limit, close to $5000 \mathrm{~km} \mathrm{~s}^{-1}$. If instead of SSRS2, we use catalogs of the new generation (deep redshift surveys obtained with $10 \mathrm{~m}$ class telescopes), it would lead to a completeness limit of $z \sim 0.7$. We model the luminosity function for nearby galaxy groups by a Schechter function with parameters $M_{\mathrm{SSRS} 2}^{*}=(-19.99 \pm 0.36)+5 \log h$ and $\alpha=-1.46 \pm 0.17$ to compute the mass to light ratio. The median value of the mass to light ratio is $360 h M_{\odot} / L_{\odot}$ (in the SSRS2 band, close to a $B$ band magnitude) and we deduce a relation between mass to light ratio and velocity dispersion $\sigma(M / L=(3.79 \pm 0.64) \sigma-(294 \pm 570))$. The more massive the group, the higher the mass to light ratio, and therefore, the larger the amount of dark matter inside the group. Another explanation is a significant stripping of the gas of the galaxies in massive groups as opposed to low mass groups. This extends to groups of galaxies the mild tendency already detected for rich clusters of galaxies. Finally, we detect a barely significant fundamental plane for these groups $\left(L \propto R_{\mathrm{Vir}}^{2.26 \pm 1.39} \times \sigma^{2.93 \pm 1.64}\right.$ for groups with more than 8 galaxies) but it is much less narrow than for clusters of galaxies.
\end{abstract}

Key words. galaxies: clusters: general - cosmology: large-scale structure of Universe

\section{Introduction}

The abundance evolution of galaxy structures is a major prediction of cosmological models (e.g. Oukbir \& Blanchard 1992, 1997; Romer et al. 2001). The more distant the structure, the stronger the constraint. Until now, however, we are limited to the study of the most massive distant structures. This is because these structures are the easiest to detect at high redshift using for example X-ray selected samples (e.g. Borgani et al. 1999; Burke et al. 1997; Ebeling et al. 2000; Gioia et al. 1990; Romer et al. 2000; Vikhlinin et al. 1998). These distant massive structures are the progenitors of the most massive $z \sim 0$ clusters. The hierarchical models predict, however, that nearby intermediate-mass clusters have formed from low mass high redshift systems as groups of galaxies. The new generation of deep galaxy surveys (VLT and Keck surveys) will be able to detect such high redshift low mass structures. This will add an element to the large-scale structure formation models.

We present in this paper a new efficient method to detect and study such structures in very large galaxy redshift

Send offprint requests to: C. Adami,

e-mail: christophe.adami@astrsp-mrs.fr samples (Sect. 2). We applied this method to the SSRS2 (da Costa et al. 1998) galaxy redshift catalog in order to quantify our detection efficiency at low redshift. We have created a catalog of groups from this survey and we have studied the properties of these structures (Sect. 3), comparing our results to literature studies (Carlberg et al. 2001; Girardi et al. 2000; Merchan et al. 2000). We conclude in Sect. 4.

\section{The method}

The structure detection we used is an hybrid method derived from the method used for the ENACS sample of galaxy clusters (e.g. Katgert et al. 1996) and from classical friend-of-friend detection methods (e.g. Huchra \& Geller 1982). The friend-of-friend algorithm is applied to a preprocessed catalog of elementary galaxy associations and not to individual galaxies. In this way we reduce the computation time by a large factor (see Sect. 3.3). The procedure consists of four steps:

- We apply a running window (with an overlap of half of the window size) to the original catalog of galaxies to divide the total area covered by the survey in several 
elementary lines of sight. The angular size $\varepsilon$ of the running window is chosen as a function of the kind of structures we are trying to detect ( $\varepsilon$ has to be close to the typical size of the structures) and as a function of the redshift of these structures;

- We search for compact galaxy redshift associations in each of these elementary beams by using a technique similar to Katgert et al. (1996). This technique is basically detecting gaps (fixed redshift separations between successive galaxies) greater than a given value (noted "gap analysis" in the following). This value is given by the typical maximal velocity dispersion of the structures we try to detect. For groups, we searched galaxies separated by more than $600 \mathrm{~km} \mathrm{~s}^{-1}$ in each elementary beam. If two galaxies are separated by more than this value, these galaxies belong to different structures. An elementary structure has more than 2 galaxies;

- We apply a friend-of-friend algorithm to merge the elementary structures into larger real structures. Two elementary structures were assumed to be connected if they were closer than $\varepsilon$. This value is an approximation of the maximal diameter of a group in real space. This allows us to generate a preliminary catalog of interesting regions with potential structures inside;

- Finally, we re-apply the gap method for each of these regions in order to detect real structures in the catalog.

\section{Application to the SSRS2 catalog: Group detection and analysis}

The SSRS2 catalog is a sample of 5369 galaxies, covering a region of $1.70 \mathrm{sr}$ of the southern sky $(b \leq-40 \mathrm{deg}$ and $\delta \leq-2.5 \mathrm{deg}$ for the southern galactic cap and $b \geq 35 \mathrm{deg}$ and $\delta \leq 0 \mathrm{deg}$ for the northern galactic cap). This cata$\log$ is $99 \%$ complete down to $m_{\mathrm{SSRS} 2}=15.5$. The $m_{\mathrm{SSRS} 2}$ magnitude is close to a $B$ magnitude (see da Costa et al. 1998). The precision of the individual galaxy redshift measurements is $\sim 40 \mathrm{~km} \mathrm{~s}^{-1}$. Detailed information on this catalog can be found in da Costa et al. (1998). We limited our analysis to the $2000<c z<20000 \mathrm{~km} \mathrm{~s}^{-1}$ range. To detect groups, we used $\varepsilon=1.55 h^{-1} \mathrm{Mpc}$. This value is an approximation of the maximal diameter of a group and gives the most similar results compared to the SSRS2 group analysis by Merchan et al. (2000). We kept only the structures with more than 4 galaxies and less than 40 galaxies (to be consistent with Merchan et al. 2000). Structures with less than or with 3 galaxies are possibly close superposition effects and not real dynamical structures (Ramella et al. 1997). Structures with more than 40 galaxies are likely to be rich clusters or diffuse elongated filaments of galaxies.

\subsection{Richness and physical extension}

The detected structures are described in Tables 1 and 2 . The mean number of galaxies per structure is $6 \pm 5$ and $90 \%$ of the structures have less than 11 galaxies. The largest group has 32 galaxies. The richest groups are the

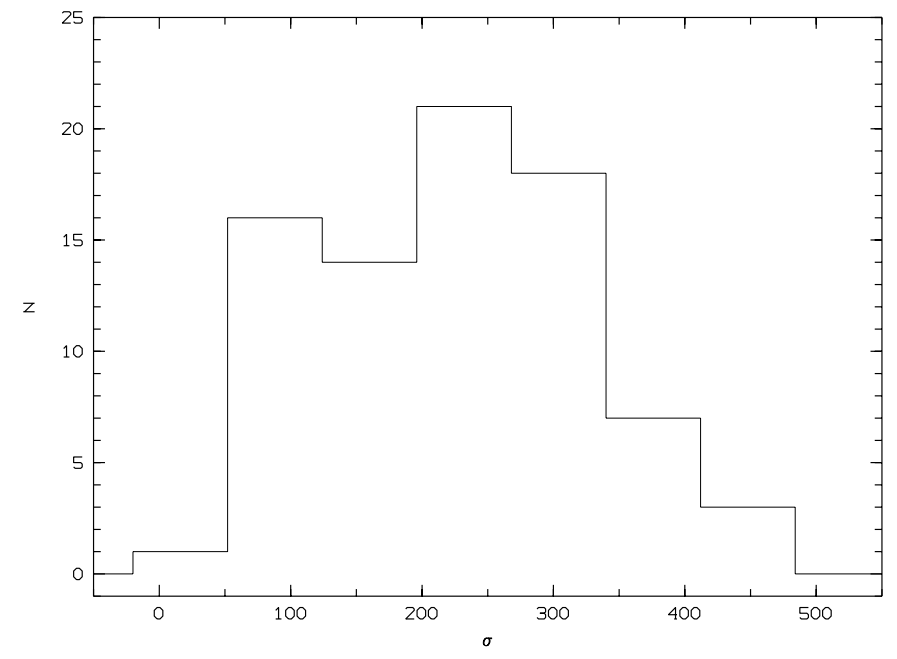

Fig. 1. Histogram of the group velocity dispersions (in $\mathrm{km} \mathrm{s}^{-1}$ ).

closer ones, in agreement with the detection of a uniform richness class of groups. This is because, due to apparent limiting magnitude, the closest groups have more galaxies brighter than the SSRS2 catalog magnitude limit.

The groups are more or less circular: they have the same angular extension in $\alpha$ (right ascension) and $\delta$ (declination). We have $\Delta \alpha-\Delta \delta=(-0.09 \pm 0.26)$ arcmin (computed with 80 groups). This means that we are not detecting systematically elongated structures such as poor filaments. The mean extension of the detected groups (computed as the dispersion of the coordinates of the galaxies of a given group) is $(0.35 \pm 0.18) h^{-1} \mathrm{Mpc}$. We have no variation of the group size as a function of the redshift, but the richest groups are also the larger ones. The groups with more than 10 galaxies have a size in the range $[0.45 ; 0.8] h^{-1} \mathrm{Mpc}$. The groups with less than 10 galaxies have a size in the range $[0.05 ; 0.65] h^{-1} \mathrm{Mpc}$. Finally, the mean virial radius $R_{\mathrm{Vir}}$ of the groups is $(0.37 \pm 0.21) h^{-1} \mathrm{Mpc}$ (in the range $[0.06 ; 0.87] h^{-1} \mathrm{Mpc}$, see Tables 1 and 2).

\subsection{Velocity dispersions and masses}

We computed velocity dispersions using a robust estimator (Beers et al. 1990). This gives a more realistic estimate of the velocity dispersion for groups with more than 10 galaxies, but still a low number, compared to classical estimators. For groups with less than 10 galaxies, there is no significant difference between this robust estimator and the classical estimator of the velocity dispersion (Lax 1985).

The mean velocity dispersion of the groups is (224 \pm 103) $\mathrm{km} \mathrm{s}^{-1}$. None of them has a velocity dispersion greater than $500 \mathrm{kms}^{-1}$ (see Fig. 1). These values are consistent with several other computations for groups (e.g. Carlberg et al. 2001 with a median velocity dispersion of $200 \mathrm{~km} \mathrm{~s}^{-1}$ ). This means that we detected no rich clusters. 


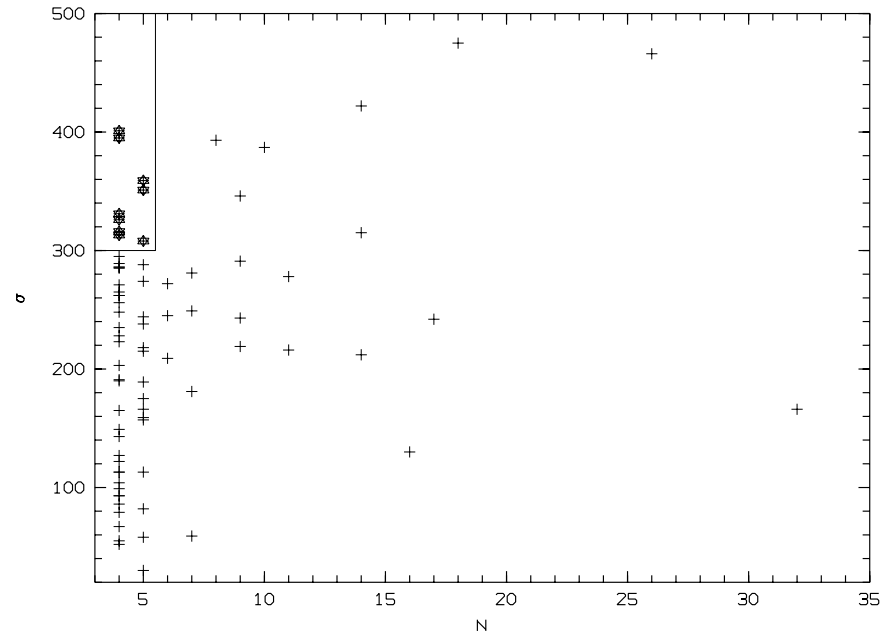

Fig. 2. Number of galaxies inside each group as a function of the velocity dispersion of the group. The stars are the groups with too low a richness compared to the velocity dispersion.

The groups with the highest velocity dispersions are also the richest (see Fig. 2) but we have no significant relation between the group mean redshift and velocity dispersion. This is, one more time, in favor of a uniform detection of groups as a function of redshift.

In order to check how many spurious groups we are detecting (groups with a large velocity dispersion, but a low number of galaxies: chance alignments), we plotted the velocity dispersion as a function of the number of galaxies inside the groups (Fig. 2). Only 10\% of the groups have less than 5 galaxies and a velocity dispersion greater than $300 \mathrm{~km} \mathrm{~s}^{-1}$. This can be assumed to be the contamination rate of our catalog.

Combining the virial radius and the velocity dispersion, we computed the mass using the standard virial mass estimator $\left.(M \propto \text { virial radius } \times \text { (velocity dispersion })^{2}\right)$ (e.g. Ramella et al. 1997). About $85 \%$ of the sample has a mass in the range $\left[5 \times 10^{12} ; 4 \times 10^{14}\right]$ solar masses and $97.5 \%$ has a mass in the range $\left[10^{12} ; 4 \times 10^{14}\right]$ solar masses. None of the groups has a mass greater than $4 \times 10^{14}$ solar masses. The mean mass is $(5.6 \pm 6.5) \times 10^{13}$ solar masses. All the masses computed by Merchan et al. (2000) are in the range $\left[5 \times 10^{12} ; 4 \times 10^{14}\right]$ solar masses. This means that we have slightly lower masses in the sample (for $15 \%$ of the sample). This is probably due to the fact that we are using robust estimators to compute velocity dispersions. Such estimators are less biased toward high values for small samples of galaxies. The velocity dispersions and masses are given in Tables 1 and 2 .

In order to estimate the mass uncertainty, we used galaxy groups with more than 10 galaxies. For these groups, a reliable error can be computed for velocity dispersion using 100 bootstrap resamplings. This is obviously a lower limit of the velocity dispersion uncertainty as this uncertainty is probably larger for smaller groups.
The $1-\sigma$ uncertainty is: $\Delta \sigma / \sigma \sim 20 \%$. The mass uncertainty can be written as:

$\Delta$ Mass $/$ Mass $=\Delta R_{\mathrm{Vir}} / R_{\mathrm{vir}}+2 \Delta \sigma / \sigma$

Assuming that the velocity dispersion uncertainty is the dominant factor, we have the $1-\sigma$ error on the mass: $\Delta$ Mass $/$ Mass $\geq 40 \%$. This large error raises the question of the validity of virial mass estimates for groups of galaxies as these structures are probably not all in equilibrium (e.g. Dos Santos \& Mamon 1999).

\subsection{Sample completeness and 2-point correlation function}

We compared our catalog with that one of Merchan et al. (2000) that used the same galaxy sample. Figure 3 shows the group redshift distribution of the two catalogs. We detect similar numbers of groups up to $c z \sim 8000 \mathrm{~km} \mathrm{~s}^{-1}$. The mean difference per bin is $5 \%$ (with redshift bins of $1250 \mathrm{~km} \mathrm{~s}^{-1}$ width). Merchan et al. detect more groups at higher redshift. However, their catalog begins to be incomplete above $5000 \mathrm{~km} \mathrm{~s}^{-1}$. This means that we have the same detection completeness level, in agreement with Press \& Schechter (1974) predictions (Merchan et al. 2000). Finally, we estimated the 2-point correlation function for the sample. Using a power law approximation of the form $\left(r / r_{0}\right)^{\gamma}$, we have $r_{0}=7.0 h^{-1} \mathrm{Mpc}$ and $\gamma=-1.79$. This is consistent at the $1 \sigma$ level with the values of Merchan et al. (2000): $r_{0}=(8.4 \pm 1.8) h^{-1} \mathrm{Mpc}$ and $\gamma=-2.0 \pm 0.7$, Girardi et al. (2000): $r_{0}=\left(8 . \pm 1\right.$.) $h^{-1} \mathrm{Mpc}$ and $\gamma=-1.9 \pm 0.7$ or Carlberg et al. (2001): $r_{0}=$ $(6.8 \pm 0.3) h^{-1} \mathrm{Mpc}$ and $\gamma=-1.8$ (fixed). We detect a positive signal up to $\sim 90 h^{-1} \mathrm{Mpc}$ (similar to the values of Merchan et al. 2000). These estimates are all in good agreement and give confidence in the detection method. The only significant difference is a lower group detection rate at the faint end of the galaxy catalog. However, this does not affect the completeness level of our group sample as we detect the same number of groups up to $c z \sim 8000 \mathrm{~km} \mathrm{~s}^{-1}$ (assuming that the Merchan et al. completeness limit is correct).

The redshift completeness limit close to $5000 \mathrm{kms}^{-1}$ is directly related to the magnitude limit of the catalog: $m_{\mathrm{SSRS} 2} \sim B \sim 15.5$. It gives an absolute magnitude of $-18+5 \log h$. This is the magnitude limit for the galaxies lying inside groups before the completeness limit. We can extrapolate this redshift completeness limit assuming the typical depth of the new generation of redshift surveys using 10 meter class telescopes (Keck and VLT). For example, the deep part of the Virmos survey should provide a redshift survey $80 \%$ complete down to $B \sim 26$, and the shallow part of this survey a $50 \%$ complete redshift catalog down to $B \sim 24$. Assuming a $k$-correction proportional to the redshift, it should provide a catalog of groups complete up to $z \sim 1.2$ for the deep catalog and $z \sim 0.7$ for the shallow catalog. This is enough to sample nearly all classes of galaxy structures up to $z \sim 0.7$. This will allow one to put strong constraints on cosmological models 
Table 1. Group sample: Col. $1(N)$ : number of galaxies inside groups, Col. $2(\alpha)$ : right ascension (in degrees), Col. $3(\delta)$ : declination (degrees), Col. 4 (z): mean redshift, Col. 5 (Obs. Lum.): observed group luminosity (luminosity before corrections: in unit of solar luminosity), Col. 6: $E(B-V)$ from Schlegel et al. (1998) at the group coordinates, Col. 7 (Ext.): extinction correction factor, Col. 8 (Comp.): completeness correction factor, Col. 9 (Tot. Lum.): group total luminosity (in unit of solar luminosity), Col. 10 (Mass): virial mass (in unit of solar mass), Col. $11(M / L)$ : mass to light ratio in the SSRS2 magnitude passband, Col. $12(\sigma)$ : velocity dispersion in $\mathrm{km} \mathrm{s}^{-1}$, Col. 13 (Rvir): virial radius in $h^{-1} \mathrm{Mpc}$.

\begin{tabular}{|c|c|c|c|c|c|c|c|c|c|c|c|c|}
\hline$N$ & $\alpha$ & $\bar{\delta}$ & $z$ & Obs. Lum. & $E(B-V)$ & Ext. & Comp. & Tot. Lum. & Mass & $M / L$ & $\sigma$ & $R_{\text {vir }}$ \\
\hline 5 & 3.2488 & -24.3504 & 0.0255 & $5.33 \mathrm{E}+10$ & 0.02220 & 1.09 & 3.20 & $1.86 \mathrm{E}+11$ & $1.90 \mathrm{E}+12$ & 10.2 & 82 & 0.13 \\
\hline 4 & 3.2868 & -7.4360 & 0.0181 & $4.54 \mathrm{E}+10$ & 0.03389 & 1.14 & 1.94 & $1.01 \mathrm{E}+11$ & $4.91 \mathrm{E}+13$ & 486.9 & 286 & 0.27 \\
\hline 4 & 5.5908 & -4.3517 & 0.0138 & $1.13 \mathrm{E}+10$ & 0.03145 & 1.13 & 1.60 & $2.04 \mathrm{E}+11$ & $2.13 \mathrm{E}+14$ & 1043.7 & 401 & 0.6 \\
\hline 5 & 6.8999 & -9.0373 & 0.0192 & $2.99 \mathrm{E}+10$ & 0.03553 & 1.15 & 2.19 & $7.56 \mathrm{E}+10$ & $1.44 \mathrm{E}+14$ & 1905.7 & 359 & 0.51 \\
\hline 4 & 7.0446 & -37.3208 & 0.0245 & $3.91 \mathrm{E}+10$ & 0.02375 & 1.10 & 3.13 & $1.34 \mathrm{E}+11$ & $4.04 \mathrm{E}+12$ & 30.1 & 86 & 0.25 \\
\hline 4 & 7.1985 & -22.9825 & 0.0268 & $7.17 \mathrm{E}+10$ & 0.01305 & 1.05 & 3.06 & $2.31 \mathrm{E}+11$ & $4.44 \mathrm{E}+12$ & 19.2 & 113 & 0.16 \\
\hline 5 & 7.3508 & -10.9007 & 0.0122 & $5.79 \mathrm{E}+10$ & 0.03334 & 1.14 & 2.15 & $1.42 \mathrm{E}+11$ & $2.45 \mathrm{E}+13$ & 172.4 & 189 & 0.31 \\
\hline 5 & 8.0602 & -3.3412 & 0.0196 & $3.70 \mathrm{E}+10$ & 0.03581 & 1.15 & 2.23 & $9.51 \mathrm{E}+10$ & $1.98 \mathrm{E}+13$ & 208.3 & 215 & 0.2 \\
\hline 4 & 8.4047 & -28.5031 & 0.0234 & $3.47 \mathrm{E}+10$ & 0.02017 & 1.08 & 2.62 & $9.86 \mathrm{E}+10$ & $2.45 \mathrm{E}+13$ & 248.4 & 149 & 0.5 \\
\hline 4 & 8.8372 & -23.7145 & 0.0128 & $1.90 \mathrm{E}+10$ & 0.01679 & 1.07 & 1.80 & $3.65 \mathrm{E}+10$ & $3.89 \mathrm{E}+12$ & 106.5 & 99 & 0.18 \\
\hline 7 & 10.6675 & -9.3056 & 0.0197 & $4.22 \mathrm{E}+10$ & 0.03604 & 1.15 & 1.89 & $9.20 \mathrm{E}+10$ & $6.88 \mathrm{E}+13$ & 747.4 & 281 & 0.4 \\
\hline 4 & 12.7327 & -13.4234 & 0.0376 & $9.85 \mathrm{E}+10$ & 0.02390 & 1.10 & 7.94 & $8.60 \mathrm{E}+11$ & $2.06 \mathrm{E}+13$ & 24.0 & 286 & 0.12 \\
\hline 4 & 14.2872 & -9.1834 & 0.0150 & $2.88 \mathrm{E}+10$ & 0.08095 & 1.38 & 2.07 & $8.22 \mathrm{E}+10$ & $1.35 \mathrm{E}+13$ & 164.2 & 113 & 0.48 \\
\hline 9 & 18.0011 & -32.3378 & 0.0192 & $1.25 \mathrm{E}+11$ & 0.02365 & 1.10 & 2.18 & $3.00 \mathrm{E}+11$ & $5.37 \mathrm{E}+13$ & 179.0 & 346 & 0.2 \\
\hline 4 & 18.0096 & -6.6967 & 0.0204 & $2.76 \mathrm{E}+10$ & 0.11385 & 1.57 & 2.37 & $1.03 \mathrm{E}+11$ & $1.10 \mathrm{E}+13$ & 107.0 & 285 & 0.06 \\
\hline 14 & 20.7478 & -35.3655 & 0.0193 & $1.23 \mathrm{E}+11$ & 0.01880 & 1.08 & 2.17 & $2.88 \mathrm{E}+11$ & $5.47 \mathrm{E}+13$ & 190.1 & 212 & 0.56 \\
\hline 5 & 20.7672 & -38.5622 & 0.0205 & $4.90 \mathrm{E}+10$ & 0.01960 & 1.08 & 2.18 & $1.15 \mathrm{E}+11$ & $7.60 \mathrm{E}+12$ & 65.8 & 159 & 0.14 \\
\hline 4 & 25.2701 & -34.4858 & 0.0128 & $1.14 \mathrm{E}+10$ & 0.01997 & 1.08 & 1.57 & $1.93 \mathrm{E}+10$ & $4.52 \mathrm{E}+12$ & 233.7 & 190 & 0.06 \\
\hline 5 & 25.3051 & -4.2930 & 0.0180 & $3.59 \mathrm{E}+10$ & 0.02266 & 1.09 & 2.05 & $8.05 \mathrm{E}+10$ & $1.92 \mathrm{E}+13$ & 238.4 & 157 & 0.36 \\
\hline 5 & 26.2856 & -13.8575 & 0.0181 & $2.74 \mathrm{E}+10$ & 0.01640 & 1.07 & 2.08 & $6.09 \mathrm{E}+10$ & $1.94 \mathrm{E}+13$ & 318.4 & 175 & 0.29 \\
\hline 4 & 28.2577 & -4.2047 & 0.0164 & $2.91 \mathrm{E}+10$ & 0.02386 & 1.10 & 1.85 & $5.92 \mathrm{E}+10$ & $1.25 \mathrm{E}+14$ & 2110.9 & 331 & 0.52 \\
\hline 4 & 28.7924 & -9.3172 & 0.0173 & $1.91 \mathrm{E}+10$ & 0.02394 & 1.10 & 1.95 & $4.10 \mathrm{E}+10$ & $1.08 \mathrm{E}+14$ & 2635.4 & 265 & 0.7 \\
\hline 4 & 29.5184 & -25.5656 & 0.0144 & $3.66 \mathrm{E}+10$ & 0.01053 & 1.04 & 1.98 & $7.56 \mathrm{E}+10$ & $2.34 \mathrm{E}+13$ & 309.5 & 256 & 0.16 \\
\hline 5 & 30.2063 & -32.1871 & 0.0183 & $4.08 \mathrm{E}+10$ & 0.01977 & 1.08 & 2.33 & $1.03 \mathrm{E}+11$ & $1.17 \mathrm{E}+14$ & 1138.0 & 288 & 0.64 \\
\hline 4 & 30.6148 & -29.3072 & 0.0169 & $3.96 \mathrm{E}+10$ & 0.01656 & 1.07 & 2.15 & $9.10 \mathrm{E}+10$ & $1.34 \mathrm{E}+14$ & 1471.9 & 289 & 0.73 \\
\hline 4 & 31.4930 & -6.8843 & 0.0169 & $1.60 \mathrm{E}+10$ & 0.01961 & 1.08 & 1.83 & $3.16 \mathrm{E}+10$ & $3.71 \mathrm{E}+13$ & 1172.2 & 271 & 0.23 \\
\hline 4 & 31.5281 & -23.1068 & 0.0178 & $5.10 \mathrm{E}+10$ & 0.01674 & 1.07 & 2.12 & $1.16 \mathrm{E}+11$ & $9.35 \mathrm{E}+13$ & 808.6 & 316 & 0.43 \\
\hline 4 & 34.8054 & -38.3532 & 0.0167 & $2.95 \mathrm{E}+10$ & 0.02016 & 1.08 & 1.83 & $5.85 \mathrm{E}+10$ & $4.24 \mathrm{E}+12$ & 72.4 & 55 & 0.64 \\
\hline 7 & 36.4329 & -11.4888 & 0.0153 & $3.37 \mathrm{E}+10$ & 0.02209 & 1.09 & 1.75 & $6.44 \mathrm{E}+10$ & $6.41 \mathrm{E}+12$ & 99.6 & 181 & 0.09 \\
\hline 9 & 39.0671 & -13.1909 & 0.0150 & $5.33 \mathrm{E}+10$ & 0.03001 & 1.13 & 1.68 & $1.01 \mathrm{E}+11$ & $4.99 \mathrm{E}+13$ & 494.9 & 243 & 0.39 \\
\hline 9 & 40.8864 & -25.7116 & 0.0229 & $1.15 \mathrm{E}+11$ & 0.01903 & 1.08 & 3.60 & $4.45 \mathrm{E}+11$ & $8.34 \mathrm{E}+13$ & 187.4 & 219 & 0.79 \\
\hline 8 & 41.4914 & -31.8348 & 0.0221 & $1.13 \mathrm{E}+11$ & 0.02305 & 1.10 & 2. & $3.17 \mathrm{E}+11$ & $1.66 \mathrm{E}+14$ & 523.7 & 393 & 0.49 \\
\hline 5 & 42.5306 & -8.9778 & 0.0178 & $2.59 \mathrm{E}+10$ & 0.02907 & 1.12 & 2.04 & $5.93 \mathrm{E}+10$ & $9.15 \mathrm{E}+13$ & 1542.9 & 274 & 0.56 \\
\hline 14 & 46.6818 & -10.4633 & 0.0155 & $8.66 \mathrm{E}+10$ & 0.07630 & 1.36 & 1.67 & $1.96 \mathrm{E}+11$ & $1.17 \mathrm{E}+14$ & 597.3 & 422 & 0.3 \\
\hline 5 & 47.4664 & -25.3934 & 0.0212 & $2.99 \mathrm{E}+10$ & 0.01680 & 1.07 & 2.33 & $7.45 \mathrm{E}+10$ & $3.07 \mathrm{E}+12$ & 41.2 & 113 & 0.11 \\
\hline 4 & 48.1498 & -7.5486 & 0.0177 & $3.10 \mathrm{E}+10$ & 0.06842 & 1.31 & 2.15 & $8.76 \mathrm{E}+10$ & $7.86 \mathrm{E}+13$ & 896.8 & 203 & 0.87 \\
\hline 10 & 49.0948 & -4.7276 & 0.0079 & $2.40 \mathrm{E}+10$ & 0.06228 & 1.28 & 1.26 & $3.87 \mathrm{E}+10$ & $8.08 \mathrm{E}+13$ & 2087.3 & 387 & 0.25 \\
\hline 4 & 52.7168 & -4.8493 & 0.0198 & $3.00 \mathrm{E}+10$ & 0.04634 & 1.20 & 2.24 & $8.10 \mathrm{E}+10$ & $8.13 \mathrm{E}+13$ & 1004.3 & 235 & 0.07 \\
\hline 4 & 53.8719 & -18.4927 & 0.0142 & $2.26 \mathrm{E}+10$ & 0.06259 & 1.28 & 2.06 & $5.97 \mathrm{E}+10$ & $1.94 \mathrm{E}+12$ & 32.5 & 67 & 0.2 \\
\hline 11 & 55.2961 & -4.5489 & 0.0138 & $1.01 \mathrm{E}+11$ & 0.06435 & 1.29 & 1.58 & $2.07 \mathrm{E}+11$ & $3.45 \mathrm{E}+13$ & 166.7 & 216 & 0.34 \\
\hline 5 & 149.4540 & -2.7081 & 0.0210 & $5.70 \mathrm{E}+10$ & 0.04201 & 1.18 & 2.13 & $1.43 \mathrm{E}+11$ & $2.49 \mathrm{E}+13$ & 173.6 & 308 & 0.12 \\
\hline 7 & 150.3229 & -6.2040 & 0.0164 & $4.93 \mathrm{E}+10$ & 0.03796 & 1.16 & 1.78 & $1.02 \mathrm{E}+11$ & $3.36 \mathrm{E}+13$ & 329.0 & 249 & 0.25 \\
\hline 5 & 151.6062 & -2.4202 & 0.0202 & $3.65 \mathrm{E}+10$ & 0.04465 & 1.19 & 2.37 & $1.03 \mathrm{E}+11$ & $2.66 \mathrm{E}+12$ & 25.8 & 58 & 0.36 \\
\hline 5 & 155.7194 & -2.3807 & 0.0186 & $5.69 \mathrm{E}+10$ & 0.04830 & 1.21 & 1.93 & $1.33 \mathrm{E}+11$ & $4.88 \mathrm{E}+13$ & 366.3 & 218 & 0.47 \\
\hline 6 & 160.6563 & -10.2180 & 0.0077 & $1.29 \mathrm{E}+10$ & 0.00910 & 1.04 & 1.38 & $1.77 \mathrm{E}+10$ & $6.25 \mathrm{E}+13$ & 3522.1 & 245 & 0.48 \\
\hline 4 & 162.5583 & -12.2600 & 0.0154 & $1.79 \mathrm{E}+10$ & 0.03559 & 1.15 & 1.86 & $3.84 \mathrm{E}+10$ & $2.77 \mathrm{E}+13$ & 721.7 & 248 & 0.21 \\
\hline 4 & 164.2253 & -9.5503 & 0.0270 & $6.25 \mathrm{E}+10$ & 0.03783 & 1.16 & 3.15 & $2.29 \mathrm{E}+11$ & $2.44 \mathrm{E}+13$ & 106.6 & 191 & 0.31 \\
\hline 17 & 165.7276 & -14.5042 & 0.0144 & $1.09 \mathrm{E}+11$ & 0.00944 & 1.04 & 1.52 & $1.66 \mathrm{E}+11$ & $2.97 \mathrm{E}+14$ & 1787.6 & 475 & 0.6 \\
\hline 5 & 168.0586 & -13.6485 & 0.0171 & $3.23 \mathrm{E}+10$ & 0.07506 & 1.35 & 1.93 & $8.42 \mathrm{E}+10$ & $1.25 \mathrm{E}+14$ & 1484.7 & 351 & 0.46 \\
\hline 4 & 170.6570 & -13.4858 & 0.0173 & $5.77 \mathrm{E}+10$ & 0.03418 & 1.15 & 1.85 & $1.22 \mathrm{E}+11$ & $7.72 \mathrm{E}+13$ & 630.9 & 244 & 0.59 \\
\hline
\end{tabular}


Table 2. Same as Table 1.

\begin{tabular}{|c|c|c|c|c|c|c|c|c|c|c|c|c|}
\hline$N$ & $\alpha$ & $\delta$ & $z$ & Raw Lum. & $E(B-V)$ & ext. & comp. & Tot. lum. & Mass & $M / L$ & $\sigma$ & $R$ vir \\
\hline 4 & 171.4233 & 11.2592 & 4 & $3 \mathrm{~F}+10$ & 0.04699 & 1.21 & 1.99 & $6.79 \mathrm{E}+10$ & $97 \mathrm{E}+13$ & 585.1 & 165 & 0.66 \\
\hline 4 & & 75 & & $\mathrm{E}+$ & & & & & $35 \mathrm{H}$ & & & .39 \\
\hline 6 & 179.4168 & 20.3306 & 0222 & $46 \mathrm{E}+10$ & 4524 & 1.20 & 2.31 & $.23 \mathrm{E}+11$ & $.77 \mathrm{E}+13$ & 386.6 & 72 & 0.29 \\
\hline 4 & & 777 & & & & & 1.67 & & 3 & 177.9 & & .56 \\
\hline 4 & & 79 & & $36 \mathrm{E}+10$ & & & & & 3 & 478.2 & & 0.4 \\
\hline 4 & & 24 & & $4.57 \mathrm{E}+10$ & & 1.07 & 1. & 10 & $.28 \mathrm{E}$ & 1450.5 & 26 & 0.54 \\
\hline 5 & & -26.9663 & & $2.64 \mathrm{E}+10$ & 7663 & 1.36 & 2.1 & $5.69 \mathrm{E}+10$ & $.71 \mathrm{E}+13$ & 476.1 & 66 & 0.45 \\
\hline 26 & & 2 & & 2.0 & & 1.25 & & 11 & 4 & 1207.8 & 66 & 0.76 \\
\hline 4 & & 36 & & & & & & & 2 & 35.5 & 223 & 0.06 \\
\hline 4 & & & & & & & & & & 76.7 & 79 & 0.15 \\
\hline 9 & & & & & & 1. & & & 9.28 & 309.1 & 291 & 0.5 \\
\hline 5 & & 3 & & 6.1 & & 1.14 & 2. & 11 & 3.86 & 238.4 & 238 & 0.31 \\
\hline 17 & & 1 & & 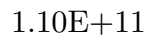 & & & & & 3 & 287.5 & & 0.46 \\
\hline 4 & & & & & & & & & & 951.6 & 262 & 0.27 \\
\hline 4 & & & & & & & & & & 25.8 & 93 & 0.14 \\
\hline 11 & & & & $7.93 \mathrm{E}+10$ & & 1.30 & & & 1.39 & 007.2 & 278 & 0.82 \\
\hline 4 & & & & $1.25 \mathrm{E}+$ & & & 1. & 1.7 & $1.56 \mathrm{l}$ & 87.6 & & 0.07 \\
\hline 4 & & & & & & & & & 1.0 & 38.8 & 228 & 0.09 \\
\hline 32 & & & & 1. & & 4 & 1 & & 2.5 & 174.4 & 166 & 0.42 \\
\hline 6 & & -27 & & $1.49 \mathrm{E}+11$ & & & & & 8.801 & 20.6 & 209 & 0.09 \\
\hline 5 & & & & & & & & & & 52.3 & 30 & 0.26 \\
\hline 4 & & -1 & & & & & & & & 22.9 & 52 & 0.44 \\
\hline 4 & & -30 & 9 & 4.6 & & 6 & & 11 & 6.94 & 593.3 & 262 & 0.46 \\
\hline 4 & & -27 & 78 & $5.87 \mathrm{E}+10$ & 950 & 1.08 & 2. & $1.74 \mathrm{E}+11$ & $1.01 \mathrm{E}+13$ & 58.1 & 122 & 0.31 \\
\hline 7 & & -21.2985 & 0.0087 & $1.61 \mathrm{E}+10$ & 2895 & 1.12 & 1. & $2.40 \mathrm{E}+10$ & $5.09 \mathrm{E}+11$ & 21.2 & 59 & 0.07 \\
\hline 4 & & & & & & & & $2.72 \mathrm{E}$ & $6.78 \mathrm{E}$ & 248.9 & 295 & 0.36 \\
\hline 16 & & -22 & & $9.25 \mathrm{E}+10$ & & & & $1.41 \mathrm{E}+11$ & $2.88 \mathrm{H}$ & 204.9 & 130 & 0.78 \\
\hline 4 & & & & $6.90 \mathrm{E}+10$ & & & & & $1.57 \mathrm{I}$ & 5.6 & 93 & 0.08 \\
\hline 14 & 356.4653 & -28.3663 & 0.0282 & $2.60 \mathrm{E}+11$ & & 1. & $3 .^{\prime}$ & $1.05 \mathrm{E}+12$ & $3.56 \mathrm{E}-$ & 33.9 & 315 & 0.16 \\
\hline 4 & 357.4948 & -29.5501 & 0.0291 & $5.24 \mathrm{E}+10$ & 0.01603 & 1.07 & 3.97 & $2.22 \mathrm{E}+11$ & $1.62 \mathrm{E}+13$ & 73.1 & 143 & 0.36 \\
\hline
\end{tabular}

(e.g. Romer et al. 2001) as well as sampling the internal structure evolution of groups (and more massive clusters) up to these redshifts.

The time required to treat these future samples with our method is very short. We used the simulations of Steve Hatton (private communication) in order to estimate these times. These simulations cover $1 \mathrm{deg}^{2}$ and reproduce an $\Omega_{m}=0.3$ and $\Omega_{\Lambda}=0.7$ Universe, including several structures as clusters or groups. We used a single $6^{\prime}$ running window for simplicity and we selected randomly different sub-samples of galaxies in this catalog. The computation times are given in Fig. 4. We see that a catalog of 150000 galaxies, comparable to the spectroscopic catalogs which will be produced by the VLT and Keck surveys, is completely analyzed in about 2 min (using an ES40 Compaq, processor EB67 at $600 \mathrm{Mhz}$ ).

\subsection{Luminosity function}

We computed the luminosity function of the group galaxies of the detected sample. The galaxy catalog is complete down to $m_{\mathrm{SSRS} 2}=15.5$ with groups up to $8000 \mathrm{~km} \mathrm{~s}^{-1}$. Limiting ourselves to groups with velocity lower than $6000 \mathrm{~km} \mathrm{~s}^{-1}$ (this velocity limit is close to the

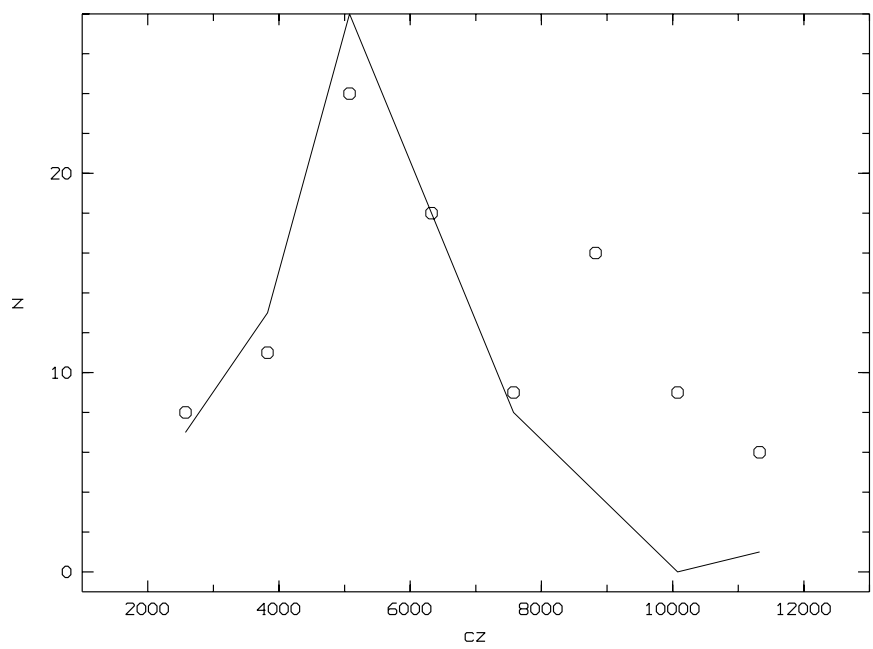

Fig. 3. Number of detected groups as a function of redshift by Merchan et al. (circles) and with our method (solid line).

completeness limit of the group sample), we have a galaxy catalog complete down to $M_{\mathrm{SSRS} 2}=-18.4+5 \log h$. Using the estimate of Zabludoff \& Mulchaey (2000), this corresponds to about $M^{*}+1.5$. We fitted a Schechter function 


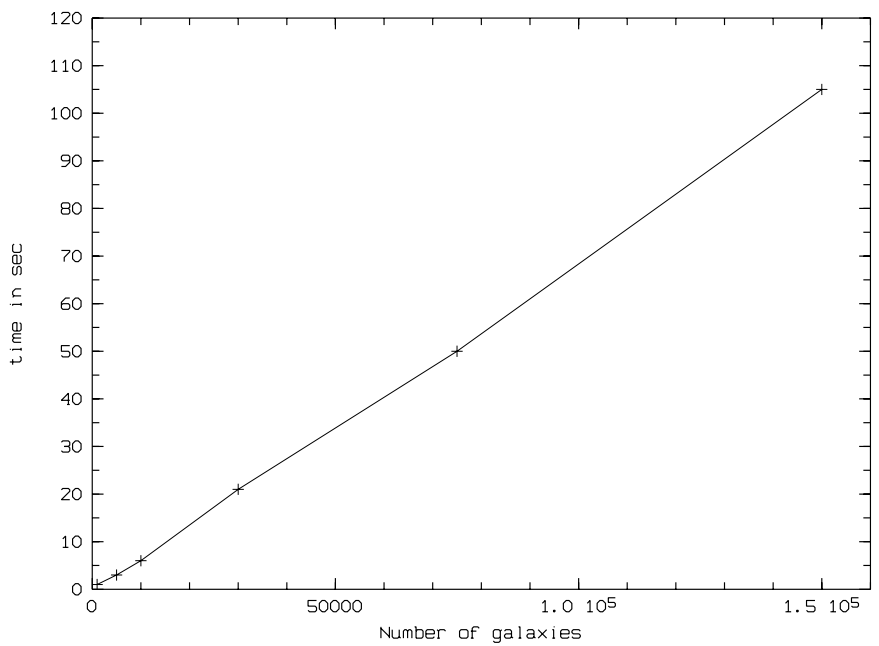

Fig. 4. Computation time (in s) for a simulated catalog of galaxies including groups and clusters, as a function of the number of galaxies inside the catalog.

on the magnitude distribution down to $M_{\mathrm{SSRS} 2}=-18.4+$ $5 \log h$ and for groups with $c z \leq 6000 \mathrm{~km} \mathrm{~s}^{-1}$ using a maximum likelihood technique (e.g. Lobo et al. 1997).

The absolute magnitudes were $k$-corrected (but this is a minor correction due to the low redshift of the sample: see Tables 1 and 2 ) using the $k$-correction $-3.5 \times z$ (Rauzy et al. 1998). We also corrected for galactic extinction using the work by Schlegel et al. (1998). The correction we applied for the magnitudes was $-4.325 \times E(B-V)$. The mean correction was $16 \%$ of the luminosity (but up to $60 \%$ for the worse cases: see Tables 1 and 2).

The best fit parameters of a Schechter function are $M^{*}=(-19.99 \pm 0.36)+5 \log h$ and $\alpha=-1.46 \pm 0.17$ (estimates with 1- $\sigma$ error $)$. This is consistent at the 1- $\sigma$ level with the estimates of Zabludoff \& Mulchaey (2000): $M_{B}^{*}=$ $(-20.1 \pm 0.4)+5 \log h$ (assuming $\left.m_{B}-m_{R} \sim 1.5\right)$ and $\alpha=-1.3 \pm 0.1$ for groups in $2800<c z<7700 \mathrm{~km} \mathrm{~s}^{-1}$. These values are also similar at the $1-\sigma$ level with the estimates of Rauzy et al. (1998) for rich clusters of galaxies.

In order to check the robustness of our estimates, we split the sample in two parts: the northern and the southern galactic cap. The southern cap gives $M^{*}=$ $(-20.26 \pm 0.69)+5 \log h$ and $\alpha=-1.61 \pm 0.47$ and the northern cap gives $M^{*}=(-19.79 \pm 0.45)+5 \log h$ and $\alpha=-1.32 \pm 0.42$. Despite the larger uncertainty, these values are still consistent, with, however, a mild tendency to have more faint galaxies in the southern galactic cap.

\subsection{Total luminosity and mass to light ratio}

We computed the total luminosity of each group summing up all the individual magnitudes (see Tables 1 and 2). We used $M_{\text {SSRS }}^{\odot}=5.53$ (approximation of $M_{B}^{\odot}$ ). We corrected these values for incompleteness due to galaxy catalog magnitude limit by using the ratio between the

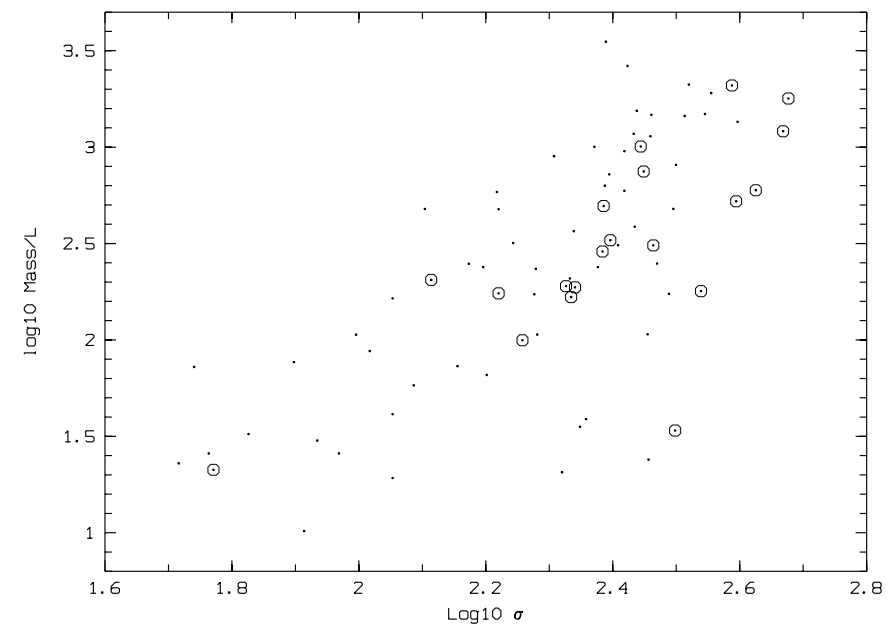

Fig. 5. Mass to light ratio as a function of the velocity dispersion for groups of galaxies detected by our method in the SSRS2 galaxy catalog. The circles are the groups with more than 8 galaxies.

luminosity function integrated from $M_{\mathrm{SSRS}}=-23+5 \log h$ to $-10+5 \log h$ and integrated between the faintest magnitude of each group and $M_{\mathrm{SSRS}}=-10+5 \log h$. This is because we assumed that the faintest galaxies were not fainter than $-10+5 \log h$. This limit has, however, a moderate influence on this correction factor. We used the luminosity function computed in the previous section with $M^{*}=(-19.99 \pm 0.36)+5 \log h$ and $\alpha=-1.46 \pm 0.17$. The mean correction was $135 \%$ of the raw group luminosity. The total luminosities and the individual completeness corrections are given in Tables 1 and 2 .

Combining estimates of the mass and of the luminosity, we computed the mass to light ratio (see Tables 1 and 2). The mean value is $550 h M_{\odot} / L_{\odot}$ and the median is $250 h M_{\odot} / L_{\odot}$. We compared these estimates with those of Carlberg et al. (2001). In the $R$ band, they computed median values in the range $[150 ; 250] h M_{\odot} / L_{\odot}$. Assuming $m_{R \odot}-m_{\mathrm{SSRS} \odot}=-1.17$ (Allen 1973) and $m_{R}-m_{\mathrm{SSRS}}=-1.5$ for galaxies in nearby structures (e.g. Katgert et al. 1998), it corresponds to median values in the range $[204 ; 340] h M_{\odot} / L_{\odot}$ in the SSRS2 magnitude passband (multiplication factor of $10^{0.4(1.5-1.17)}$ ). This is in good agreement with our estimates. We found a relation between the mass to light ratio and the velocity dispersion $\sigma$ for our sample (see Fig. 5 and Table 3).

These relations are qualitatively similar to the relation we found between rich cluster velocity dispersion and cluster mass to light ratio (Adami et al. 1998): $M / L=$ $(0.58 \pm 0.17) \sigma-(12 \pm 236)$. The slope is, however, significantly steeper for groups than for clusters. The slope of these relations is also similar to that of Carlberg et al. (2001) which is close to 3.5. The more massive the group (or the cluster), the higher the mass to light ratio, and therefore, the larger the amount of dark matter inside 
Table 3. Various relations between $M / L$ ratio and velocity dispersions for our sample. The first column describes the selection (number of galaxies per group or cluster sample of Adami et al. 1998), the second column is the slope of the linear relation and the third column is the constant term of the linear relation. The fourth column is the dispersion (in percents) of the relations for groups.

\begin{tabular}{rrrr}
\hline Selection & slope & constant & dispersion \\
\hline \hline All SSRS2 groups & $3.79 \pm 0.64$ & $294 \pm 570$ & $8 \%$ \\
\hline $\begin{array}{r}\text { SSRS2 groups with } \\
\text { more than } 7 \text { galaxies }\end{array}$ & $3.57 \pm 0.89$ & $462 \pm 431$ & $6 \%$ \\
\hline $\begin{array}{r}\text { SSRS2 groups with } \\
\text { more than 8 galaxies }\end{array}$ & $3.90 \pm 1.17$ & $579 \pm 470$ & $4 \%$ \\
\hline Cluster sample & $0.58 \pm 0.17$ & $12 \pm 236$ & - \\
\hline
\end{tabular}

the group (or cluster). Another explanation would be a very efficient stripping of the gas of the galaxies in rich groups (as opposed to very low mass groups). This would be due to a denser intra group medium or a more efficient tidal stripping due to the higher number of galaxies (e.g. Dos Santos \& Mamon 1999) in rich groups. It would lower the star formation rate in galaxies, inducing a higher mass to light ratio for the richest groups (see also Carlberg et al. 2001). The dispersion of the relation is also smaller for richer groups. This is probably due to a better computation of the velocity dispersion due to the larger number of available galaxies.

\subsection{A fundamental plane for groups of galaxies?}

Schaeffer et al. (1993) or Adami et al. (1998) have shown, using optical data, that clusters of galaxies populate a well defined area in the [total luminosity: $L$, characteristic radius of a beta profile: $R_{\mathrm{c}}$, velocity dispersion: $\sigma$ ] space. Adami et al. (1998) found the following relation:

$L \propto R_{\mathrm{c}}^{1.19} \times \sigma^{0.91}$ with a dispersion of the relation of $5 \%$. Using the virial radius instead of the characteristic radius of a beta profile would give:

$L \propto R_{\mathrm{Vir}}^{1.51} \times \sigma^{1.07}$.

This relation, called the fundamental plane, is also detected for elliptical galaxies, but with different coefficients (see Adami et al. 1998 for a comparison). This is interpreted as the different relaxation state of elliptical galaxies and clusters of galaxies. We searched for the same kind of relation in the groups we detected. Using all the groups, we found no relation between $L, R_{\mathrm{Vir}}$ and $\sigma$. Using only groups with more than 8 galaxies, we found: $L \propto R_{\mathrm{Vir}}^{2.26 \pm 1.39} \times \sigma^{2.93 \pm 1.64}$ with a dispersion of $58 \%$.

The coefficients are consistent with those of clusters of galaxies, but the uncertainties are very large, and the intrinsic dispersion of the relation is 10 times larger than for clusters. We conclude that a mean relation between $L$, $R_{\mathrm{Vir}}$ and $\sigma$ probably exists for groups, but this relation is much less well defined than for rich clusters. Moreover, poor groups (less than 7 galaxies) probably have too large a velocity dispersion uncertainty to allow any detection of this relation.

\section{Conclusion}

We demonstrated that we are able to detect similar populations of groups with our method compared to classical friend-of-friend algorithms. The groups detected with our method have the same physical properties as those detected by Merchan et al. (2000): nearly the same mass distribution (most of our groups are in the range $\left[5 \times 10^{12} ; 4 \times 10^{14}\right]$ solar masses), same abundances up to $c z \sim 8000 \mathrm{~km} \mathrm{~s}^{-1}$ (and, therefore, same completeness limit: $\sim 5000 \mathrm{~km} \mathrm{~s}^{-1}$ and abundances in agreement with Press \& Schechter models), and same 2-point correlation function (modeled by a power law: $\left(r / r_{0}\right)^{\gamma}$ with $r_{0}=7 h^{-1} \mathrm{Mpc}$ and $\left.\gamma=-1.79\right)$.

This redshift completeness limit close to $5000 \mathrm{~km} \mathrm{~s}^{-1}$ is directly related to the magnitude limit of the catalog: $m_{\mathrm{SSRS} 2} \sim B \sim 15.5$. Extrapolating these limits assuming the typical depth of the new generation of redshift surveys using 10 meter class telescopes (Keck and VLT) leads to a redshift completeness limit of $z \sim 0.7$ for the groups in these samples.

We found for the SSRS2 catalog a similar luminosity function for nearby group galaxies to that of Zabludoff et al. (2000): $M^{*}=(-19.99 \pm 0.36)+5 \log h$ and $\alpha=$ $-1.46 \pm 0.17$. We computed a similar mass to light ratio compared to Carlberg et al. (2001) (median value of $250 h M_{\odot} / L_{\odot}$ in the B SSRS2 magnitude passband) and we deduced a similar relation between the mass to light ratio and velocity dispersion $(M / L=(3.79 \pm 0.64) \sigma-$ $(294 \pm 570))$. This relation is qualitatively similar to that detected for rich clusters of galaxies (Adami et al. 1998), but with a significantly steeper slope. The more massive the group (or the cluster), the higher the mass to light ratio, and therefore, the larger the amount of dark matter inside the group (and cluster). Another explanation is a significant stripping of the gas of the galaxies in rich groups as opposed to poorer groups. Finally, we detected a fundamental plane for these groups $\left(L \propto R_{\mathrm{Vir}}^{2.26 \pm 1.39} \times \sigma^{2.93 \pm 1.64}\right.$ for groups with more than 8 galaxies) but that is much less narrow and barely significant compared to clusters of galaxies. We conclude that a mean relation between $L$, $R_{\text {Vir }}$ and $\sigma$ probably exists for groups, but this relation is much less well defined compared to clusters.

Acknowledgements. The authors thank the referee for useful comments, all the VIRMOS team in France and Italy for help and F. Durret for a careful reading of the manuscript.

\section{References}

Adami, C., Mazure, A., Biviano, A., et al. 1998, A\&A, 331, 493 
Allen, C. 1973, Astrophysical Quantities, University of London (the Athlone Press)

Beers, T., Flyn, K., \& Gebhardt, K. 1990, AJ, 100, 32

Borgani, S., Rosati, P., Tozzi, P., \& Norman, C. 1999, ApJ, 517,40

Burke, D., Collins, C., Sharples, R., et al. 1997, ApJ, 488, L83

Carlberg, R., Yee, H., Morris, S., et al. 2001, ApJ, 552, 427

da Costa, L., Willmer, C., Pellegrini, P., et al. 1998, AJ, 116, 1

Ebeling, H., Jones, L., Perlman, E., et al. 2000, ApJ, 534, 133

Gioia, I., Henry, J., Maccacaro, T., et al. 1990, ApJ, 356, L35

Girardi, M., Boschin, W., \& da Costa, L. 2000, A\&A, 353, 57

Huchra, J., \& Geller, M. 1982, ApJ, 257, 423

Katgert, P., Mazure, A., den Hartog, R., et al. 1998, A\&AS, 129,399

Katgert, P., Mazure, A., Perea, J., et al. 1996, A\&A, 310, 8

Lax, D. 1985, J. Am. Stat. Assoc., 80, 736
Lobo, C., Biviano, A., Durret, F., et al. 1997, A\&A, 317, 385 Merchan, M., Maia, M., \& Lambas, D. 2000, ApJ, 545, 26 Oukbir, J., \& Blanchard, A. 1997, A\&A, 317, 1

Oukbir, J., \& Blanchard, A. 1992, A\&A, 262, L21

Press, W., \& Schechter, P. 1974, ApJ, 187, 425

Ramella, M., Pisani, A., \& Geller, M. 1997, AJ, 113, 483

Rauzy, S., Adami, C., \& Mazure, A. 1998, A\&A, 337, 31

Romer, A., Viana, P., Liddle, A., \& Mann, R. 2001, ApJ, 547, 594

Romer, A., Nichol, R., Holden, B., et al. 2000, ApJS, 126, 209

Dos Santos, S., \& Mamon, G. 1999, A\&A, 352, 1

Schaeffer, R., Maurogordato, S., Cappi, A., \& Bernardeau, F. 1993, MNRAS, 263, L21

Schlegel, D., Finkbeiner, D., \& Davis, M. 1998, ApJ, 500, 525

Vikhlinin, A., Mc Namara, B., Forman, W., et al. 1998, ApJ 498, L21

Zabludoff, A., \& Mulchaey, J. 2000, A\&A, 331, 493 